\title{
Edaravone for amyotrophic lateral sclerosis: barriers to access and lifeboat ethics
}

\author{
Ari Breiner MD MSc, Lorne Zinman MD MSc, Pierre R. Bourque MD
}

- Cite as: CMAJ 2020 March 23;192:E319-20. doi: 10.1503/cmaj.191236

A myotrophic lateral sclerosis (ALS) is a fatal neurodegenerative condition that results in progressive paralysis of limb and bulbar muscles, with an average life expectancy of 3-5 years after diagnosis. Until 2017, riluzole was the only approved medication for patients with ALS, offering a limited survival benefit of about 3-5 months. ${ }^{1}$ In May 2017, a small clinical trial involving patients with grade 1 or 2 ALS in Japan found that edaravone slowed disease progression in early stage ALS. ${ }^{2}$ The results of this study were met with elation by many patients with ALS, desperate for additional effective treatment options. However, access to edaravone for many patients with ALS in Canada has been fraught with barriers over the last few years, and limited supply has resulted in ethical dilemmas for clinicians. We present evidence of the efficacy of edaravone, describe the history of access to the drug in Canada and consider what is needed to ensure that Canadian patients with ALS who are most likely to benefit have access to the drug.

Since the introduction of riluzole in 1994, numerous clinical trials of proposed treatments for ALS have failed. Edaravone is a free radical scavenger that has been available in Japan since 2003 for the treatment of acute stroke, ${ }^{3}$ but also showed promise in mouse models for ALS. An initial randomized controlled trial (RCT) in patients with ALS did not show benefit for all patients, although a subgroup of patients in the earliest disease phase did benefit. ${ }^{4} \mathrm{~A}$ subsequent trial compared edaravone plus riluzole versus riluzole alone in patients with early-stage ALS who were free of substantial respiratory muscle weakness and who had progressed over a 3-month observation period. ${ }^{2}$ This study showed a $33 \%$ slowing in the rate of disease progression ${ }^{2}$ over a 6-month treatment period. Although the effect of edaravone on survival could not be reliably assessed because of the short duration of the trial, the $33 \%$ slowing provided by edaravone may be extrapolated to a survival benefit of 4-5 months compared with riluzole alone. ${ }^{5}$

After publication of the trial results, ${ }^{2}$ edaravone was approved in the United States by the U.S. Food and Drug Administration in May 2017 for all patients with ALS. Although the drug was marketed widely in the US, it was initially unavailable in Canada. However, some Canadian patients with ALS acquired a generic form of edaravone through direct contact with Japanese neurology clinics. ${ }^{6}$ Legal justification was provided through the Canadian

\section{KEY POINTS}

- A Japanese randomized controlled trial showed edavarone - a free radical scavenger previously used to treat acute stroke - to be effective in slowing progression of amyotrophic lateral sclerosis (ALS) for people with early-stage disease.

- Despite its unavailability in Canada, patients legally imported the drug under provisions of the Canadian Food and Drugs Act and then had to find ways to receive intravenous administration.

- Edavarone was approved by Health Canada in October 2018, and its manufacturer established a compassionate use program to provide no-cost edaravone access to 210 Canadian patients through provincial Special Access Programmes, which created an ethical dilemma for clinics.

- Edavarone's manufacturer and the Patented Medicine Prices Review Board have not yet reached an agreement on the list price, which means that access remains limited, mostly through select private payers.

- The drug should be publicly reimbursed for patients with ALS who meet the inclusion criteria of the clinical trial that showed the drug's efficacy (about $10 \%$ of patients with ALS) without delay.

Food and Drugs Act, ${ }^{7}$ which allows for the personal importation of medications not commercially available in Canada if solely for personal use. Canadian patients cited "right-to-try" legislation to justify acquiring this medication that was not approved in Canada. ${ }^{8}$ Clinics treating ALS were overwhelmed by patients requesting assistance with intravenous administration of an unfamiliar, unapproved medication with packaging information in Japanese (edaravone is not approved for the treatment of patients with stroke in Canada). Hospital administration and nursing staff were uncertain whether to permit the infusion of edaravone in hospital because of concerns regarding insurance coverage if patients had serious adverse events. Some patients self-administered the medication, which is administered 10 days per month, in their homes with the assistance of family or friends. In addition, some patients with more advanced ALS, despite not being expected to benefit from edaravone based on evidence, exerted pressure on physicians to assist with infusions.

Approval for edaravone from Health Canada was sought in April 2018 and granted in October 2018. However, additional challenges emerged. While under review by Health Canada, the 
manufacturer established a compassionate use program to provide no-cost edaravone access to some Canadian patients with ALS through provincial Special Access Programmes. Because access was limited to 210 Canadians, ${ }^{9}$ each clinic had to select the patients who would be offered edaravone from among about 3000 Canadians living with ALS. This program created a "lifeboat ethics" dilemma, whereby physicians treating ALS became responsible for the distribution of a scarce resource among seemingly equally deserving patients with a terminal disease. Practices varied: some clinics set up lotteries to distribute edaravone while others dissuaded patients from receiving edaravone until it became more widely available. Some clinics adhered rigidly to the inclusion/exclusion criteria of the clinical trial when deciding who would be eligible, whereas others were more liberal. Simultaneously, patients excluded from the Special Access Programmes continued to purchase edaravone privately resulting in differential access based on personal finances.

Access to edaravone has varied among countries. Japan, South Korea, the US and Switzerland have approved the medication with relatively broad access. The European Medicines Authority requested that a controlled clinical trial of edaravone show an effect on survival before approval will be granted. The Canadian Agency for Drugs and Technology in Health reanalyzed the manufacturer's economic analysis and calculated a cost greater than $\$ 1.9$ million per quality-adjusted life-year, based on an annual drug cost of $\$ 185000 .^{10}$ The agency suggested funding the medication only for patients who meet the original study inclusion criteria and conditional on price reduction. ${ }^{10}$ However, the manufacturer and the Patented Medicine Prices Review Board have been unable to reach an agreement on the list price. Therefore, only limited distribution, mostly through select private payers, has occurred.

As clinicians caring for patients with ALS, we must prioritize the needs of patients living with this rapidly fatal and debilitating neurodegenerative disease. However, we must also adhere to scientific evidence, and we agree that cost-effectiveness is a valid consideration when making decisions about publicly funded treatments. Given that Health Canada has concluded that the drug is efficacious, we believe Canadian publicly funded drug plans and the manufacturer must negotiate a national price that accounts for edaravone's limited therapeutic efficacy but also fairly compensates for drug development costs. Because cost is a substantial concern, we suggest that the drug should be publicly reimbursed for those patients with ALS who meet the inclusion criteria of the clinical trial that showed the drug's efficacy (about $10 \%$ of patients with ALS) without delay. If we fail to offer effective new therapies to these patients after positive clinical trials, there will be little motivation for researchers or patients to work toward finding better treatments for this devastating disease.

\section{References}

1. Bensimon G, Lacomblez L, Meininger V. A controlled trial of riluzole in amyotrophic lateral sclerosis. ALS/Riluzole Study Group. N Engl J Med 1994;330:585-91.

2. Writing Group; Edaravone (MCl-186) ALS 19 Study Group. Safety and efficacy of edaravone in well defined patients with amyotrophic lateral sclerosis: a randomised, double-blind, placebo-controlled trial. Lancet Neurol 2017;16:505-12.

3. Edaravone Acute Infarction Study Group. Effect of a novel free radical scavenger, edaravone ( $\mathrm{MCl}-186)$, on acute brain infarction. Randomized, placebo-controlled, double-blind study at multicenters. Cerebrovasc Dis 2003;15:222-9.

4. Abe K, Itoyama Y, Sobue G, et al.; Edaravone ALS Study Group. Confirmatory double-blind, parallel-group, placebo-controlled study of efficacy and safety of edaravone (MCl-186) in amyotrophic lateral sclerosis patients. Amyotroph Lateral Scler Frontotemporal Degener 2014;15:610-7.

5. CADTH Common Drug Review: pharmacoeconomic review report. Edaravone (Radicava) (Mitsubishi Tanabe Pharma Corporation): Indication - for the treatment of amyotrophic lateral sclerosis (ALS). Ottawa: Canadian Agency for Drugs and Technologies in Health; 2019.

6. Curwin L. Terri Bishop is living with ALS but still embraces the good in each day. Truro News 2018 May 25.

7. Food and Drugs Act (R.S.C., 1985, c. F-27). Available: https://laws-lois.justice. gc.ca/eng/acts/f-27/ (accessed 2020 Mar. 2).

8. Laucius J. 'I'm going to die anyway': Should terminally ill patients in Canada get 'the right to try'? Ottawa Citizen 2016 Dec. 2. Available: https://ottawacitizen.com/news/ national/should-dying-patients-have-the-right-to-try-unapproved-drugs-a-battle-is -brewing (accessed 2020 Mar. 2).

9. Access to therapies: Radicava (edaravone) update. Toronto: Amyotrophic Lateral Sclerosis Society of Canada; 2019. Available: www.als.ca/blogs/access-to-therapies -radicava-edaravone-update/ (accessed 2020 Feb. 19).

10. CADTH Canadian drug expert committee recommendation: Edaravone (Radicava Mitsubishi Tanabe Pharma Corporation) - indication: for the treatment of amyotrophic lateral sclerosis. Ottawa: Canadian Agency for Drugs and Technologies in Health; 2019.

Competing interests: Ari Breiner has served as site principal investigator for trials for amyotrophic lateral sclerosis funded by Mallinkrodt, Cytokinetics and the University of Calgary. He has also received research grant funding from CIDP/GBS Foundation International, Grifols and Muscular Dystropy Canada, and honoraria from CSL, Allergan, Pfizer and Akcea. Ari Breiner and Lorne Zinman have received honoraria from Mitsubishi Tanabe Pharma. Lorne Zinman has also received an honorarium from Biogen. No other competing interests were declared.

This article has been peer reviewed.

Affiliations: Division of Neurology (Breiner, Bourque), Department of Medicine, The Ottawa Hospital and University of Ottawa; Ottawa Hospital Research Institute (Breiner, Bourque), Ottawa, Ont.; Sunnybrook Research Institute (Zinman), Hurvitz Brain Sciences Program, Sunnybrook Health Sciences Centre; Division of Neurology (Zinman), Department of Medicine, University of Toronto, Toronto, Ont.

Contributors: Ari Breiner and Lorne Zinman were joint primary authors. All of the authors contributed equally to the manuscript. All of the authors conceived the work, drafted the manuscript, analyzed and interpreted the data, reviewed the manuscript critically for important intellectual content, gave final approval of the version to be pubished and agreed to be accountable for all aspects of the work.

Correspondence to: Ari Breiner, abreiner@toh.ca 